\title{
Associated production of the Higgs boson and a single top quark in the littlest Higgs model at Large Hadron Collier
}

\author{
Shuo Yang \\ Institute of Theoretical Physics, Chinese Academy of Sciences, Beijing 100190, China*
}

\begin{abstract}
In the context of the littlest Higgs model, we study the associated production of the Higgs boson and a top quark (th production) at the CERN Large Hadron Collider (LHC). The cross sections for s-channel, t-channel processes and the relative correction for the total cross section are presented. In a part of parameter space, the cross sections can be distinctly deviated from the predictions of Standard Model. We also investigate the signal and backgrounds for the th production at the LHC. However, due to the large QCD backgrounds, it is not very hopeful to directly observe the th signal in most of the parameter space of LH model. It is found that with $30 \mathrm{fb}^{-1}$ of integrated luminosity, in a narrow range of the parameter space $c=0.8$ and $f \leq 1.62 \mathrm{TeV}$, a statistical significance of $3 \sigma$ can be achieved. More than $30 \mathrm{fb}^{-1}$ luminosity will enhance the significance and the possibility of detecting the signal.
\end{abstract}

PACS numbers:

*Electronic address: shuoyang@itp.ac.cn 


\section{INTRODUCTION}

For the Standard Model (SM) Higgs boson, the main discovery modes at hadron colliders are the associated production of $W h$ or $Z h$ [1], the vector boson fusion processes [2], the gluon-gluon fusion mode [3], and the Higgs associated production with heavy top quark pairs [4] or bottom quark pairs [5]. Compared with these modes, the cross section for the production of the Higgs boson associated with a single top quark is small [6, 7, 8]. (th production Feynman diagrams are shown in Fig.1-Fig.3 .) However, measurements of the total rates for $W h$ and $t \bar{t} h$ processes only test the Higgs coupling to $W$ and the Yukawa coupling to top. The th production contains the important relative phase information of the couplings of the Higgs to $W$ and to top, and th production is sensitive to some new physics [7, 8]. As a class candidate of physics beyond SM, little Higgs models [9, 10, 11, 12, 13, 14, 15] predict new bosons, fermions and scalars. These new particles will contribute considerably to Higgs production. The Higgs phenomena in little Higgs models have been extensively studied in the literature [16, 17]. In this paper, we study the th production at the LHC in the frame of the littlest Higgs model.

The rest of this paper is organized as follows. In Sec. II we briefly describe the general features of little Higgs models and then focus on the littlest Higgs model. The Feynman rules and formulas relevant to our computation are also listed in this section. In Sec. III, we study th production in the littlest Higgs model at the LHC. Finally, we give our conclusions in Sec. IV.

\section{LITTLEST HIGGS MODEL}

In this section we firstly introduce the general features of little Higgs models and then describe the littlest Higgs model focusing on particle content and the couplings relevant to our computation.

The major motivation of little Higgs theory [9] is to cure the hierarchy problem of SM. Little Higgs models suppose that Higgs boson is a Nambu-Goldstone boson (NGB) of some approximate global symmetry and avoid one-loop quadratically divergent through collective

breaking mechanism [9]. The collective symmetry breaking means that when one interaction breaks some of the global symmetries, it still exits unbroken global symmetry ensuring the 
Higgs's identity as an exact NGB. The symmetries protecting Higgs is explicitly broken only when two or more couplings in the lagrangian are present. In this way, the collective symmetry breaking protects the Higgs mass from receiving quadratically divergent radiative corrections at one-loop. The Coleman-Weinberg potential generates the Higgs potential and triggers electroweak symmetry breaking (EWSB). The implementation of collective symmetry breaking results in the prediction of new gauge bosons, new fermions and new scalars at $\mathrm{TeV}$ scale. These new particles will be produced at the LHC and contribute derivations to SM processes.

As a realistic implementation of little Higgs idea, the littlest Higgs (LH) model [11] base on a $S U(5) / S O(5)$ coset. The $S U(5)$ global symmetry is spontaneously broken down to $S O(5)$ global symmetry via a vacuum expectation value (VEV) of order $f$, simultaneously, and the gauge group $[S U(2) \times U(1)]_{1} \times[S U(2) \times U(1)]_{2}$ of $S U(5)$ is broken down to its diagonal subgroup. The breaks result in new heavy bosons $W_{H}^{ \pm}, Z_{H}, B_{H}$ and new scalar triplet $\Phi$, which acquire masses of order $f$. In order to implement the collective symmetry breaking mechanism in top sector, LH model also introduces a pair vector-like quarks. After EWSB, the physical states in top sector are the top quark $t$ and the heavy quark $T$. We list the Feynman rules and formulas [18, 19] relevant to our computation as below where the terms of order $\left(\frac{v}{f}\right)^{2}$ has been neglected:

$$
\begin{aligned}
& V_{W_{H}^{+\mu} \bar{u} d}=-i \frac{g}{\sqrt{2}} \gamma_{\mu} V_{u d} \frac{c}{s} P_{L} \quad V_{W_{H}^{+\mu} \overline{t b}}=-i \frac{g}{\sqrt{2}} \gamma_{\mu} V_{t b} \frac{c}{s} P_{L} \\
& V_{W_{H}^{+\mu} \bar{T} b}=-i \frac{g}{\sqrt{2}} \gamma_{\mu} V_{t b} \frac{c}{s} \frac{v}{f} \frac{x_{\lambda}^{2}}{x_{\lambda}^{2}+1} P_{L} \quad V_{W \bar{T} b}=i \frac{g}{\sqrt{2}} \frac{v}{f} \frac{x_{\lambda}^{2}}{x_{\lambda}^{2}+1} \gamma_{\mu} V_{t b} P_{L} \\
& V_{W_{H}^{+\mu} W_{H}^{-\mu} h}=-\frac{i}{2} g^{2} v g_{\mu \nu} \quad V_{W_{H}^{+\mu} W^{-\mu} h}=-\frac{i}{2} g^{2} \frac{\left(c^{2}-s^{2}\right)}{2 s c} v g_{\mu \nu} \\
& V_{H t \bar{T}}=-i x_{\lambda} \frac{m_{t}}{v} P_{L} \\
& M_{W_{H}}=\frac{g f}{2 s c} \quad \Gamma_{W_{H}}=\frac{\alpha_{e}}{96 \sin \theta_{W}^{2}}\left[\frac{96 c^{2}}{s^{2}}+\frac{\cos \theta_{W}^{2}\left(c^{2}-s^{2}\right)^{2}}{s^{2}-c^{2}}\right]
\end{aligned}
$$




$$
M_{T}=\left(x_{\lambda}+x_{\lambda}^{-1}\right) \frac{m_{t}}{v} f \quad \Gamma_{T}=\frac{1}{8 \pi} \frac{\lambda_{1}^{2}}{\lambda_{2}^{2}}\left(\frac{m_{t}}{\nu}\right)^{2} M_{T}
$$

In these formulas, $c\left(s=\sqrt{1-c^{2}}\right)$ is the mixing parameter between the $S U(2)_{1}$ and $S U(2)_{2}$ gauge bosons, $f$ is the new symmetry breaking scale, and $x_{\lambda}=\lambda_{1} / \lambda_{2}$, where $\lambda_{1}$ and $\lambda_{2}$ are Yukawa coupling parameters in top sector. The couplings of $\Phi$ to fermions are suppressed by $1 / f$ and the contribution of $\Phi$ to th production can be safely ignored, so we do not list the coupling of $\Phi$ here.
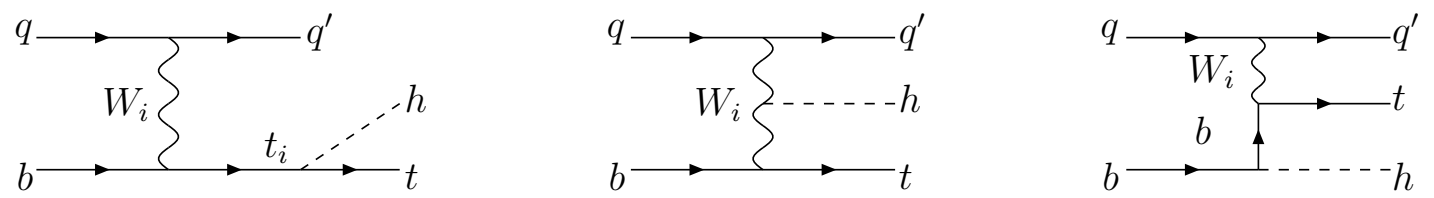

FIG. 1: Feynman diagrams for th production in t-channel process.
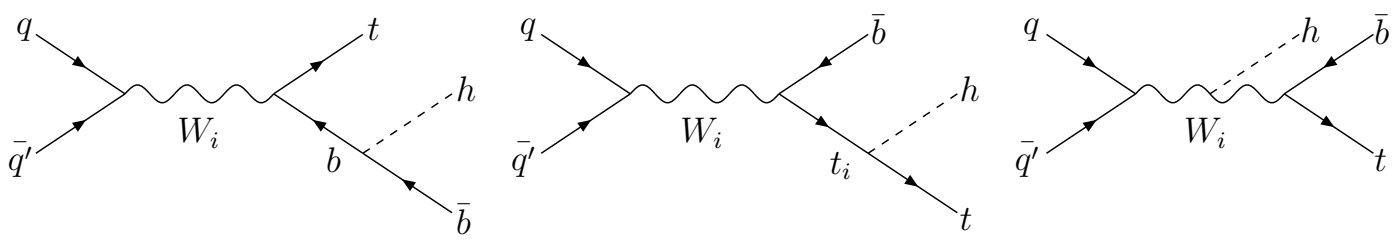

FIG. 2: Feynman diagrams for th production in s-channel process.
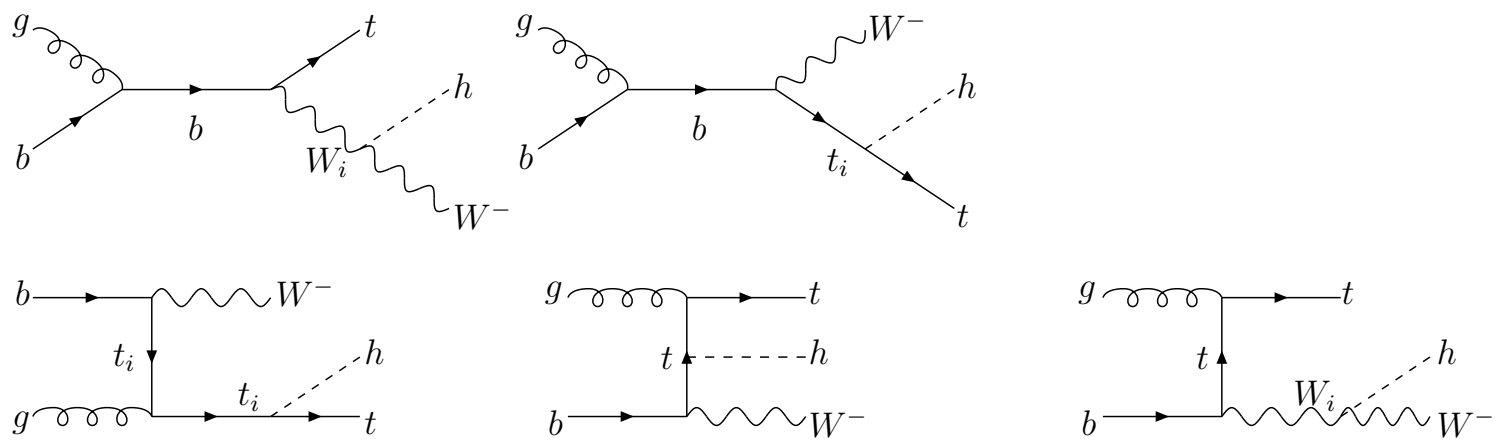

FIG. 3: Feynman diagrams for th production in $\mathrm{W}$-associated process. 


\section{III. th PRODUCTION IN THE LITTLEST HIGGS MODEL AT THE LHC}

As shown as in Fig.1 - Fig.3, the Higgs in association with a top quark can be produced at the LHC in t-channel, s-channel and W-associated process. In these Feynman diagrams, $t_{i}$ represents $t$ for $\mathrm{SM}$ and represents $t$ and $T$ for $\mathrm{LH}$ model, $W_{i}$ represents $W$ for $\mathrm{SM}$ and represents $W$ and $W_{H}$ for $\mathrm{LH}$ model. In SM, the t-channel process has the largest cross section and the s-channel has the smallest cross section. However, in LH model both the cross sections in s-channel and t-channel can dominate over the $\mathrm{W}$-associated process in a part of the parameter space as shown below.

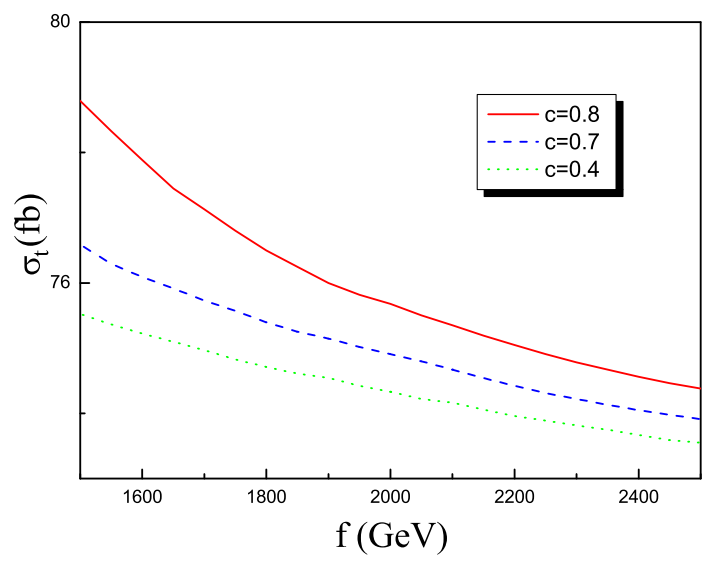

FIG. 4: Cross section for t-channel th production as a function of the parameter $f$ for $\mathrm{c}=0.4$ (dot line), $\mathrm{c}=0.7$ (dash line), $\mathrm{c}=0.8$ (solid line).

In our computation (We compute th production including both top quark and antitop production.), we have used Madgraph [20] package and CTEQ6L [21] parton distribution functions with the factorization scale $Q=m_{h}$. The SM input parameters relevant to our computation are taken as $m_{t}=174.2 \mathrm{GeV}[22], m_{h}=120 \mathrm{GeV}, m_{Z}=91.1876 \mathrm{GeV}$, $\sin ^{2} \theta_{W}=0.2315, \alpha_{e}\left(M_{Z}\right)=1 / 128.8$ and $\alpha_{s}\left(M_{Z}\right)=0.1176$ [23].

In LH model, the new interactions will contribute the th production and cause deviation from the cross section of SM. Considering the constraints of the electroweak precision data on LH model [24], we assumed $x_{\lambda}=1,1.5 \mathrm{TeV} \leq f \leq 2.5 \mathrm{TeV}$ and $0.4 \leq c \leq 0.8$. A low $f$ will result in a light $B_{H}$ which is disfavored by $Z^{\prime}$ limit from Tevatron and precision electroweak constrains. This can be resolved by considering alternative embeddings of the two $\mathrm{U}(1)$ generators or only gauging a $\mathrm{U}(1)$ group, i.e. the gauge group is $S U(2)_{1} \times S U(2)_{2} \times U(1)_{Y}$ 


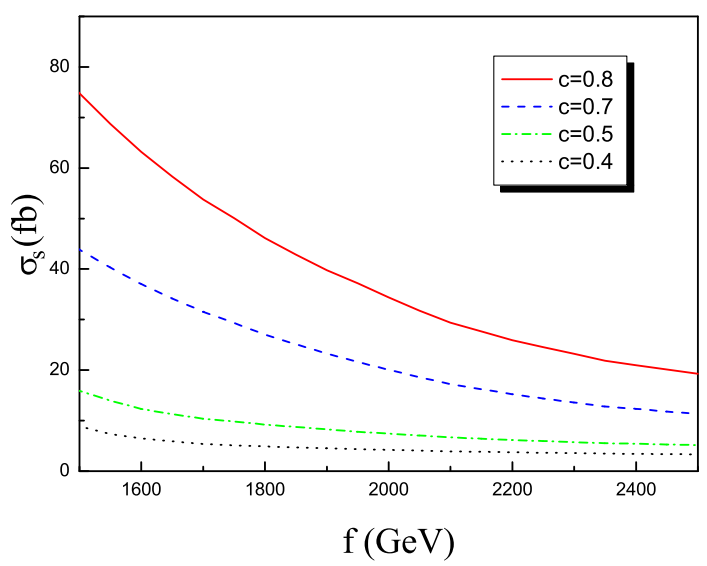

FIG. 5: Cross section for s-channel th production as a function of the parameter $f$ for $\mathrm{c}=0.4$ (dot line), $\mathrm{c}=0.5$ (dash dot line), $\mathrm{c}=0.7$ (dash line), $\mathrm{c}=0.8$ (solid line).

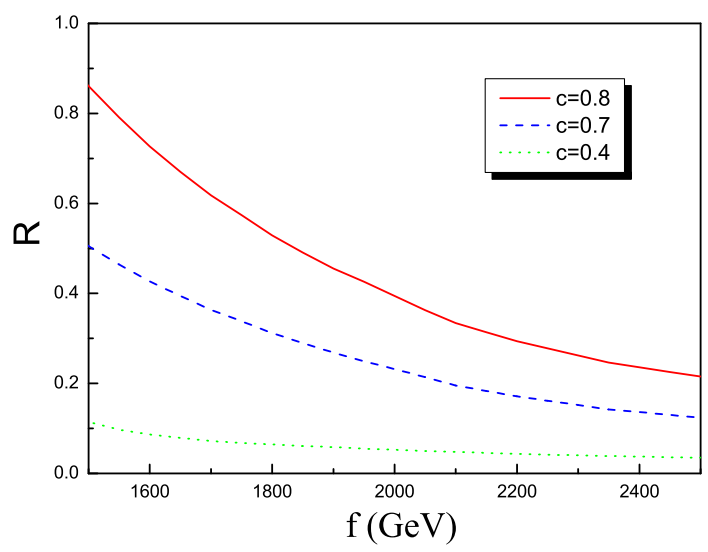

FIG. 6: Relative correction $\mathrm{R}$ of total th production as a function of the scale parameter $f$ for $\mathrm{c}=0.4($ dot line $), \mathrm{c}=0.7$ (dash line), $\mathrm{c}=0.8$ (solid line).

[24]. Fig.4 and Fig.5 show the cross sections as a function of the scale parameter $f$ for different value of mixing parameter $c$ for t-channel and s-channel th production, respectively. The computation results are very sensitive to the value of $c$, which because the coupling of $W_{H}$ to fermions are in proportion to $\frac{c}{s}$ and the cross section for relevant diagrams will be sensitive to $\left(\frac{c}{s}\right)^{4}$. In LH model, the cross section both in s-channel process and t-channel process can reach the level of $70 \mathrm{fb}$ in a part of parameter space. The cross section for $\mathrm{W}$-associated channel is not sensitive to the variation of parameters and in most of the 
parameter space the deviation from the SM prediction is small, so we don't show the cross section for $\mathrm{W}$-associated th production. Different from the case in SM where the t-channel process has the largest cross section and s-channel has the smallest one, in LH model both the cross sections in s-channel and t-channel can dominate over the $\mathrm{W}$-associated process in a part of the parameter space. This is mainly because the couplings of $W_{H} W h$ and Tth are suppressed by mixing parameters as shown in Feynman rules, so the contributes from new particles are very weak in W-associated channel. In t-channel and s-channel, the new contributes mainly come from $W_{H} q q^{\prime}$ and $W_{H} W_{H} h$ which are not suppressed. However, the effects from new particles become weak and the cross sections of th production converge to the values of SM as $f$ increase and c decrease. In order to describe the deviation of total th cross section from the SM prediction, we define a relative correction function

$$
R=\frac{\sigma_{L H}-\sigma_{S M}}{\sigma_{S M}}
$$

and show the $R$ as a function of $f$ for $c=0.4, c=0.7$ and $c=0.8$ in Fig.6. As shown in Fig.6, it is found that in most of the parameter space, the th cross section in LH model is larger than the prediction of SM which will enhance the detecting possibility in this mode.

Now we further consider the signature of th production at the LHC. In order to provide a efficient lepton trigger the semi-leptonical decay of top is considered, and the decay of $h \rightarrow b \bar{b}$ is required for the large branching ratio. In this case for t-channel mode the signal is $3 b+l^{ \pm}+\not p_{T}+1$ forward jet and for s-channel the signal is $4 b+l^{ \pm}+\not p_{T}$. However, ref. [8] has pointed that it is difficult to extract the signal $3 b+l^{ \pm}+\not p_{T}+1$ forward jet from the large backgrounds and the hopeful signal for t-channel is $4 b+l^{ \pm}+\not p_{T}+1$ forward jet where the additional $b$ comes from the splitting of virtual gluons into $b \bar{b}$ pairs. From a phenomenological point the difference between s-channel $4 \mathrm{~b}$ signal and t-channel $4 \mathrm{~b}$ signal is that all the jets are high $p_{T}$ central jets in s-channel while t-channel signal has a forward jet and a low $p_{T} b$-jet. We may separate them by rejecting or tagging a forward jet. But the s-channel signal will always be accompanied by a jet coming from QCD radiation. If we consider

[1] In our calculation, we ignored the corrections to branching ratio of $h \rightarrow b \bar{b}(B R(h \rightarrow b \bar{b}))$ in LH model. Because in LH model couplings of fermions and gauge bosons of SM to the Higgs are only shifted by an order of $(v / f)^{2}$, and only decay widths of $h \rightarrow \gamma \gamma, h \rightarrow g g$ and $h \rightarrow \gamma Z$ are modified due to new particles in loops. For a light Higgs, there is no new decay channel and the dominant decay channel is still $h \rightarrow b \bar{b}$. So the change of $B R(h \rightarrow b \bar{b})$ is not distinct in most of the parameter space and the change converges to zero as f increases. Detailed analysis of Higgs decay in LH model have been studied in Ref. [17]. 
the s-channel signal and t-channel signal separately, each of them will be the important backgrounds for the other. So, in this paper, we consider the signal is $4 b+l^{ \pm}+\not p_{T}+1$ jet which include t-channel events and s-channel events with a radiated jet. We study the signal and backgrounds in a idealized case that the top is reconstructed with $100 \%$ efficiency, so the $b$ coming from top decay can be separated from other $b$ quarks.

For the $4 b+l^{ \pm}+\not p_{T}+1$ jet signal, $t Z \bar{b} j$ with $Z$ decay to $b \bar{b}$ pair, $t b \bar{b} \bar{b} j$ are two of the main backgrounds. The process $t \bar{t} b \bar{b} \rightarrow W^{+} W^{-} b \bar{b} b \bar{b} \rightarrow j j l v b \bar{b} b \bar{b}$ contributes to backgrounds when one of the jets from $\mathrm{W}$ decay is missed or in the case that one of the jets from $\mathrm{W}$ decay is mistagged as a $b$-jet and another jet ( including $b$ ) is missed. Due to the large rate of $t \bar{t} j$, when both $c$ and $s$ quarks from the hadronically decaying $\mathrm{W}$ are mistagged, this process also contributes to the backgrounds.

Because for many events in our signal the final particles from new heavy particles' contributions are more energetic and with high- $p_{T}$ than the events in SM, So we raise the minimum $p_{T}^{b}$ to $20 \mathrm{GeV}$ and loose the forward jet constrain compared with the cuts in Ref.[8]. The cuts are shown as below:

$$
\begin{aligned}
& p_{T}^{b}>20 \mathrm{GeV},\left|\eta_{b}\right|<2.5 \\
& p_{l, v}^{T}>20 \mathrm{GeV},\left|\eta_{l}\right|<2.5 \\
& p_{j}^{T}>30 \mathrm{GeV},\left|\eta_{j}\right|<5, \Delta R_{i j}>0.4
\end{aligned}
$$

At least one $b \bar{b}$ pair $\left|m_{b \bar{b}}-m_{h}\right|<22 \mathrm{GeV}$.

All $b \bar{b}$ pairs $\min m_{b \bar{b}}>90 \mathrm{GeV}$

At least one $b \bar{b}$ pair $\left|m_{b \bar{b}}-m_{h}\right|<22 \mathrm{GeV}$ means that we require at least one $b \bar{b}$ pair (not including the $b$ that reconstructs the top ) accord with $\left|m_{b \bar{b}}-m_{h}\right|<22 \mathrm{GeV}$. All $b \bar{b}$ pairs min $m_{b \bar{b}}>90 \mathrm{GeV}$ means that the invariant mass of all $b$ pairs ( not including the $b$ that reconstructs the top ) is required in this range.

TABLE I: Cross section and events with $30 \mathrm{fb}^{-1}$ of integrated luminosity for the signal and backgrounds for the th production. For the signal, the parameter values $c=0.8, f=1.6 \mathrm{TeV}, x_{\lambda}=1$ are chosen.

\begin{tabular}{llllll}
\hline & Signal & $t Z \bar{b} j$ & $t b \bar{b} \bar{b}$ & $t \bar{t} b \bar{b}$ & $t \bar{t} j$ \\
\hline cross section $\sigma(\mathrm{fb})$ & 0.482 & 0.054 & 0.039 & 0.61 & 0 \\
events with $30 \mathrm{fb}^{-1}$ & 14.5 & 1.6 & 1.2 & 18.3 & 0 \\
\hline
\end{tabular}


At luminosity $30 \mathrm{fb}^{-1}$, only in a very narrow part of the parameter space $c=0.8$ and $f<1.62 \mathrm{TeV}$ of LH model, we can achieve the statistical significance of $3 \sigma$. We list the cross section and events with $30 \mathrm{fb}^{-1}$ which pass the cuts for the signal and the important backgrounds in table I, in which for the signal the parameter values $c=0.8, f=1.6 \mathrm{TeV}$, $x_{\lambda}=1$ are taken. The $b$-tagging efficiency $\epsilon_{b}=60 \%$, lepton-tagging efficiency $\epsilon_{l}=90 \%$ and the mistag probability $\epsilon_{c}=10 \%, \epsilon_{s}=1 \%$ have been included in computation.

\section{CONCLUSION}

In the frame of the littlest Higgs model we calculate the production of the Higgs bosons associated a top quark at the LHC. Due to the new interactions of new gauge boson $W_{H}$ and new fermion $T$ with Higgs boson, these new particles can contribute to th production cross section. We find that in a large part of the parameter space the cross section of $t h$ in s-channel and t-channel production can deviate largely from the SM prediction. Different from the case in SM, in LH model both the cross sections in s-channel and t-channel are larger than that in $\mathrm{W}$-associated process in a part of the parameter space. We also consider the signal $4 b+l^{ \pm}+\not p_{T}+1$ jet and backgrounds for the th production. However, due to the large QCD backgrounds, it is not very hopeful to observe the th signal in most of the parameter space of LH model. It is found that with $30 \mathrm{fb}^{-1}$ of integrated luminosity, in a narrow range of the parameter space $c=0.8$ and $f \leq 1.62 \mathrm{TeV}$, a statistical significance of $3 \sigma$ can be achieved. More than $30 \mathrm{fb}^{-1}$ luminosity will enhance the significance and the possibility of detecting the signal.

\section{Acknowledgments}

The author would like to thank Chun Liu, Jin Min Yang, Chong-Xing Yue and Lei Wang for useful discussions. This work was supported in part by the National Science Foundation of China under Grant No. 90503002 and the Project of Knowledge Innovation Program 
(PKIP) of Chinese Academy of Sciences, Grant No. KJCX2.YW.W10.

[1] S. L. Glashow, D. Nanopoulos, A. Yildiz, Phys. Rev. D 18, 1724(1978); J. Finjord, G. Girardi, P. Sorba, Phys. Lett. B 89, 99 (1979); E. Eichten, I. Hinchliffe, K. Lane, C. Quigg, Rev. Mod. Phys. 56, 579, (1984)

[2] D. R. T. Jones and S. T. Petcov. Phys. Lett. B 84, 440, (1979); R.N. Cahn and S. Dawson, Phys. Lett. B 136, 196 (1984) and Erratum-ibid.B 138, 464 (1984)

[3] H. Georgi, S. Glashow, M. Machacek and D. Nanopoulos, Phys. Rev. Lett. 40, 692 (1978)

[4] R. Raito and W. W. Wada, Phys. Rev. D 19, 941 (1979); Z. Kunszt, Nucl. Phys. B 247, 339 (1984); A. S. Bagdasaryan et al., Sov. J. Nucl. Phys 46, 315 (1987); J. Ng and P. Zakarauskas, Phys. Rev. D 29, 876 (1984)

[5] R. M. Barnett, H. E. haber and D. E. Soper, Nucl. Phys. B 306, 697 (1988); F. I. Olness, W.-K. Tung, Nucl. Phys. B 308, 813 (1988); D. A. Dicus, S. Willenbrock, Phys. Rev. D 39, $751(1989)$

[6] J. L. Diaz-Cruz and O. A. Sampayo, Phys. Lett. B 276, 211 (1992); W. J. Stirling, D. J. Summers, Phys. Lett. B 283, 411 (1992); A. Ballestrero, E. Maina, Phys. Lett. B 299, 312 (1993)

[7] G. Bordes, B. van Eijk, Phys. Lett. B 299, 315 (1993); T. Tait, C.-P. Yuan, Phys. Rev. D 63, $014018(2001)$

[8] F. Maltoni, K. Paul, T. Stelzer, S. Willenbrock, Phys. Rev. D 64, 090423 (2001)

[9] N. Arkani-Hamed, A. G. Cohen, H. Georgi, Phys. Lett. B 513, 232 (2001); N. Arkani-Hamed, A. G. Cohen, T. Gregoire and J. G. Wacker, JHEP 0208, 020 (2002)

[10] N. Arkani-Hamed, A. G. Cohen, E. Katz, A. E. Nelson, T. Gregoire, J. G. Wacker, JHEP 0208, $021(2002)$

[11] N. Arkani-Hamed, A. G. Cohen, E. Katz, A. E. Nelson, JHEP 0207, 034 (2002)

[12] D. E. Kaplan, M. Schmaltz, JHEP 0310, 039 (2003); M. Schmaltz, JHEP 0408, 056 (2004)

[13] H. C. Cheng, I. Low, JHEP 0309, 051 (2003); JHEP 0408, 061 (2004); I. Low, JHEP 0410, 067 (2004); H. C. Cheng, I. Low, L. T. Wang, Phys. Rev. D 74, 055001 (2006)

[14] For recent reviews see: M. Schmaltz, D. Tucker-Smith, Ann. Rev. Nucl. Part. Sci 55, 299 $(2005)$ 
[15] M. Perelstein, Prog. Part. Nucl. Phys 58, 247 (2007)

[16] J.-J. Liu, W.-G. Ma, G. Li, R.-Y. Zhang and H.-S. Hou, Phys. Rev. D 70, 015001 (2004); C.-X. Yue, W. Wang, F. Zhang Commun. Theor. Phys. 45, 511 (2006); C.-X. Yue, F. Zhang, S. Yang, Y.-B. Sun Commun. Theor. Phys. 46, 891 (2006); C.-R. Chen, K. Tobe, C.-P. Yuan, Phys. Lett. B 640, 263 (2006); C. O. Dib, R. Rosenfeld and A. Zerwekh JHEP 0605, 074 (2006); L. Wang, W.-Y. Wang, J. M. Yang, H.-J. Zhang Phys. Rev. D 75, 074006 (2007); P. Kai, R.-Y. Zhang, W.-G. Ma, H. Sun, L. Han, Y. Jiang Phys. Rev. D 76, 015012 (2007); L. Wang, W.-Y. Wang, J. M. Yang, H. -J. Zhang Phys. Rev. D 76, 017702 (2007); K. Cheung, J. Song, Phys. Rev. D 76, 035007 (2007); Y.-B. Liu, L.-L. Du, X.-L. Wang, J.Phys.G 33, 577 (2007)

[17] C. Dib, R. Rosenfeld, A. Zerwekh, hep-ph/0302068; G. A. Gonzalez-Sprinberg, R. Martinez, J.-Alexis Rodriguez, Phys. Phys. Rev. D 71, 035003 (2005)

[18] T. Han, H. E. Logan, B. McElrath, L. T. Wang, Phys. Rev. D 67, 095004 (2003)

[19] T. Han, H. E. Logan, L. T. Wang, JHEP 0601, 099 (2006)

[20] T. Stelzer and W.-F. Long, Comput. Phys. Commun. 81, 357 (1994)

[21] J. Pumplin et al. (CTEQ Collaboration), JHEP 02, 032 (2006).

[22] The Tevatron Electroweak Working Group, For the CDF and D $\varnothing$ Collabrations, hep-ex/0604053; hep-ex/0603039

[23] W.-M. Yao et al., J. Phys. G 33, 1 (2006)

[24] C.Csaki, J. Hubisz, G. D. Kribs, P. Meade, J. Terning, Phys. Rev. D 67, 115002 (2003); J. L. Hewett, F. J. Petriello, T. G. Rizzo, JHEP 0310, 062 (2003); C. Csaki, J. Hubisz, G. D. Kribs, P. Meade, J. Terning, Phys. Rev. D 68, 035009 (2003); M. C. Chen, S. Dawson, Phys. Rev. D 70, 015003 (2004); M. C. Chen et al., Mod. Phys. Lett. A 21, 621 (2006); W. Kilian, J. Reuter, Phys. Rev. D 70, 015004 (2004) 